\title{
Research on EMG Signal Extraction Based on Biomimetic Hand Wanfen $\mathrm{Xu}^{1, \mathrm{a}}$, Ying Sun ${ }^{1, \mathrm{~b}}$ and Cuiqiao $\mathrm{Li}^{1, \mathrm{c}}$ \\ ${ }^{1}$ Wuhan University of Science and Technology, China \\ aone_fen@foxmail.com, bsunying65@wust.edu.cn, ${ }^{c} 1137410667 @ q q . c o m$
}

Keywords: EMG; Bionic hand; Signal feature extraction

\begin{abstract}
EMG is a signal which could reflect the information of human body's movement in residual limb muscle group. As a source of information, it could control the bionic prosthesis to complete the multi-degree of freedom of action. EMG prosthesis controlled the bionic prosthesis through the body's own physiological signal. It is remarkable significant for the disabled users that if the bionic prosthesis could understand the movement of the disabled users will. The purpose of the EMG signal acquisition is driving a bionic hand which could help the hand disabilities to achieve grasping, pushing, taking, holding etc. EMG prosthetic is a complex system, which involving multidisciplinary disciplines. And this paper mainly introduces the method of EMG extraction.
\end{abstract}

\section{Introduction}

Many years ago, the intelligent bionic hand relied on the external drivers, and recently, it had turn to relied on the EMG signals, EGG signals drive, etc. Intelligent bionic hand's research has mainly focus on the bionic structure of the decorative design and also focus on the EMG signal control source of the pattern recognition design. Germany Ottobock company had produced the first generation of myoelectric bionic hand in 1992, it could recognized the "grasp" and "loose" in two degrees of freedom. And the recognition accuracy rate can be as high as $80 \%$. ARTS Lab of SSA's Cyber hand project and the Italian INIL/RTR center designed of a false hand which is a multi-finger less hand-driven, the use of EMG signals to control the multi-joint fingers, and it could be achieved more accurate crawling function with the simple command [1]. The foreign typical bionic hand such as the Washington University developed exoskeleton robot which can imitate the normal seven freestyle of the action; The European bionic hand could calculate the acquisition of the relevant orders and simple interaction with a perception function; The bionic hand in British had the able to identify the motor instruction issues by the brain, and also had the able to perform the complex finger movement. The development of EMG prosthetic hand is also gradually following up in China, in recent years, domestic universities have also made some progress in the study of bionic hand to promote the EMG bionic hand progress. 


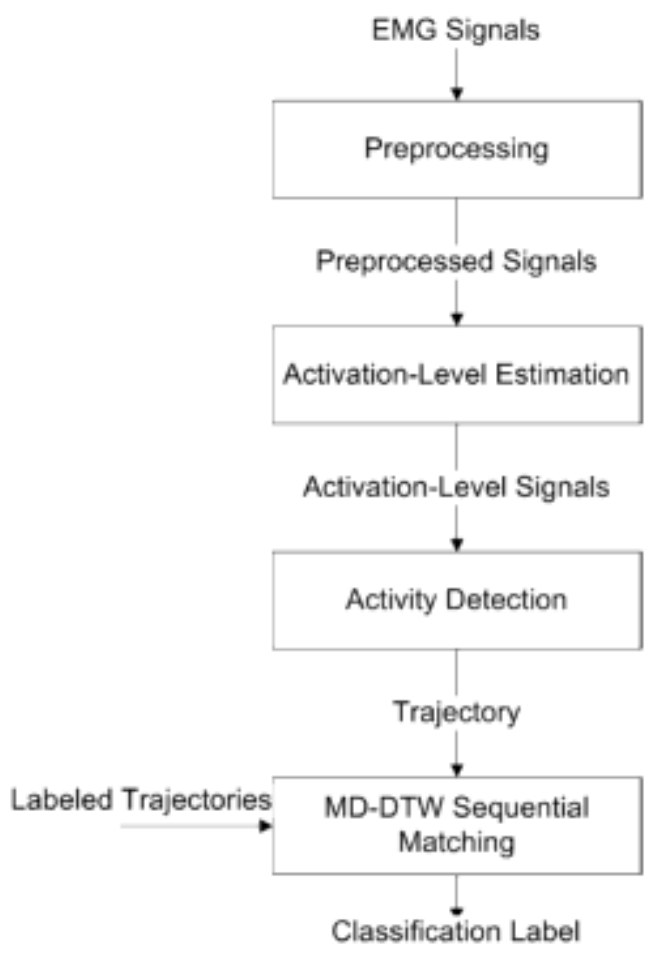

Figure 1. Finite The EMG Signal Extraction Process

\section{Feature Extraction of EMG Signals}

The extraction of effective EMG signals is significant for the analysis of EMG signals. With the development of detection technology and the computer technology, quantitative analysis and in-depth study of EMG has become possible [2]. EMG feature extraction can be summed up into four categories.

Time Domain Analysis Method. Traditional method of electromyography is to take the EMG signal as a random signal whose mean value is zero and the variance varies with the signal intensity. Time domain feature extraction is relatively simple, so the time domain analysis method in the field of EMG signal applications have been more widely used.

Integral Electromyography. The characteristic of the EMG signal were be result approximately zero due to the EMG signal as a mean zero random signal and the different between the signal can not be characterized. The mean value of the obtained signal will be greater than zero if the absolute value of the EMG signal which is the time domain analysis of the electromyography method [3]. It is defined as follows:

$$
\mathrm{X}_{\text {iemg }}=\frac{1}{\mathrm{~N}} \sum_{\mathrm{i}=0}^{\mathrm{N}-1}\left|\mathrm{x}_{(\mathrm{i})}\right|
$$

Where $x(i),(i=0,1,2 \ldots, N-1)$ is a time series of length $\mathrm{N}$.

Zero Crossing. EMG signal is derived from the electrical pulse which sent by the central nervous system. The intensity of the EMG signal is also related to the frequenvy of the electrical pulse. Therefore, the zero-crossing point is sometimes used as a feature of the EMG signal. The eigenvalue is defined as follows:

$$
\mathrm{ZC}=\sum_{\mathrm{i}=0}^{\mathrm{N}-1} \operatorname{sgn}\left(-\mathrm{x}_{(\mathrm{i})} \mathrm{x}_{(\mathrm{i}+1)}\right)
$$

Variance. It is equal to zero when the EMG signal is not treated, and take the absolute value of 
the original EMG signals, the mean value of the signal is equal to its integral EMG value. The variance would be different due to the different mean value of the electrical signals.

(1) Directly seek the original RMG signal variance [4].

$$
[\mathrm{VAR}]_{1}=\frac{1}{\mathrm{~N}-1} \sum_{\mathrm{i}=0}^{\mathrm{N}-1} \mathrm{x}_{(\mathrm{i})}^{2}
$$

(2) Take absolute value to the original EMG signal, and seek the obtained signal variance.

$$
[\mathrm{VAR}]_{2}=\frac{1}{\mathrm{~N}-1} \sum_{\mathrm{i}=0}^{\mathrm{N}-1}\left(\mathrm{x}_{(\mathrm{i})}-\mathrm{x}_{\text {iemg }}\right)^{2}
$$

Where $x_{\text {iemg }}$ is the integral electromyography of the EMG signal.

Timing Model of EMG Signal. In 1975, Graupe introduced the time series analysis technique into the EMG signal, identified the different actions by establishing the ARMA model for the EMG signal. In 1982, they improved it to AR model, which is a linear second-order moment smooth model, it is more suitable for short data analysis, and convenient operation.

EMG Histogram. The EMG signal deviates greatly from its baseline due to muscle contraction, the samples of EMG signals in different amplitudes can be used as an effective feature. In order to extract the feature, it must be set a threshold, and dived the distance between positive and negative thresholds into different amplitude segments, set the number of different amplitudes of EMG as the feature [5]. The threshold level and the number of segments are determined by experimentally.

\section{Frequency Domain Analysis Method}

When the size of muscle contractile force changes slightly, the integral electromyography, variance and other time domain became unstable, but the frequency domain description is relative stable. The stability of the power spectrum of the EMG signal directly leads to the domain feature relatively stable which extracted by the power spectrum. Therefore, the extracted frequency domain feature facilitates the subsequent EMG signal pattern recognition. The average power frequency is defined as follows:

$$
\mathrm{f}_{\text {mean }}=\int_{0}^{+\infty} \mathrm{fP}(\mathrm{f}) \mathrm{df} / \int_{0}^{+\infty} \mathrm{P}(\mathrm{f}) \mathrm{df}
$$

The median frequency is defined as follows:

$$
\int_{0}^{\mathrm{f}_{\mathrm{mf}}} \mathrm{P}(\mathrm{f}) \mathrm{df}=\int_{\mathrm{f}_{\mathrm{mf}}}^{+\infty} \mathrm{P}(\mathrm{f}) \mathrm{df}=\frac{1}{2} \int_{0}^{+\infty} \mathrm{P}(\mathrm{f}) \mathrm{df}
$$

It was found that the conduction rate of the EMG signal was linearly proportional to the characteristic frequency of the EMG signals. In the process of muscle fatigue, the median frequency as a function of time, and use the function to quantify the degree of muscle fatigue to compensation. Regardless the joint whether flexion or not, the normalized median frequency of the active and antagonistic muscle would decreases with time. And the correlation coefficient of the median frequency in the two EMG signals increases with the increase of the contractile force [6].

\section{Time-Frequency Analysis Method}

The traditional Fourier transform can only characterize the global frequency characteristics of the signal, and could not provide the frequency information of the signal in any time domain. But the time-frequency analysis method can combine the time domain and the frequency domain, and analyze the signal in time and frequency domain, and thus has received extensive attention.

Short-Term Fourier Transform. The short-term Fourier transform was proposed by Gabor in 1946, and the idea of its transformation is that the framework of Fourier transform, the nonstationary 
signal is regarded as a series of short-term stationary signal superposition, its short time through the time domain on the window to get.

Wigner-Ville Transform. The Wigner-Willy distribution is the distribution of signal energy in time and frequency, and it has many excellent features. For example, the domain identity, inversion characteristics, etc., which makes it in the non-stationary EMG signal processing has considerable potential [7].

Choi-Williams Distribution. The Choi-Williams distribution is shown below

$$
\mathrm{D}(\mathrm{t}, \omega)=\frac{1}{2 \pi} \int_{-\infty}^{+\infty} \int_{-\infty}^{+\infty} \int_{-\infty}^{+\infty} \mathrm{x}(\mathrm{u}+\tau / 2) \mathrm{x}^{*}(\mathrm{u}-\tau / 2)
$$

$\mathrm{g}(\theta, \tau) \mathrm{e}^{-\mathrm{j}(\theta t+\omega \tau-\mathrm{u} \theta)} \mathrm{dud} \tau \mathrm{d} \theta$

Wavelet Transform. The wavelet transform was proposed by the French geologists MorletJ and Grossmann A in 1984 for seismic data analysis. They found that the traditional Fourier transform is difficult to meet the requirements, thus introducing the concept of wavelet. Wavelet theory and its ideas come from Fourier analysis, which is a new development of Fourier analysis. The coefficients of the traditional Fourier series can not reflect the local characteristics of the signal, while the wavelet transform coefficients give the information of this local performance, which has a properties in both the time domain and the frequency domain [8]. The orthogonal function system in wavelet analysis is to select the appropriate basic wavelet, change the shift and scale to generate wavelet. Wavelet analysis uses short windows at high frequencies and wide windows at low frequencies, so that to provide a way for real-time processing of EMG signals.

\section{Nonlinear Dynamic Analysis Method}

The EMG signals are a kind of complex nonlinear signal, so the nonlinear method can be used in it. Many scholars have established a variety of models for the EMG signal, such as linear system model, centralized parameter model, non-stationary model and bipolar model, etc [9]. These models are linear models, and the EMG signals are actually a complex nonlinear signal, so they can be using non-linear methods. The nonlinear dynamical parameters applied in the field of feature extraction which is mainly related to four dimensions: correlation dimension, Lyapunov index, entropy and complexity.

\section{Conclusion}

The feature extraction of EMG signals have their own characteristics, the specific application in the bionic hand is based on the actual situation. Because of the instability and susceptible to interferences, the method of time domain is limited its limitations in the field of EMG signal processing applications. And the stability of EMG signal's spectrum making the frequency domain became more popular. The time-frequency analysis which represented by wavelet transform has great potential in the analysis of EMG signals. And it is being more and more valuable. The complexity of EMG signal was determines that it can not make full use of information if it was only one method [10]. And the rapid development of computer technology has provides the possibility in which of various technologies for EMG signal processing.

\section{References}

[1] X Zhang, X Chen, Y Li, et al: A Framework for Hand Gesture Recognition Based on Accelerometer and EMG Sensors. Systems And Humans, Vol. 41 (2011) No.1, p.1064-1079

[2] X.X Li, O.W Samuel, X Zhang, et al: A motion-classification strategy based on sEMG-EGG signal combination for upper-limb amputees. Journal of NeuroEngineering and Rehabilitation, Vol.14 (2017) No.2, p.1-13

[3] Imteyaz Ahamd, F Ansari, U.K. Dey: A Review of EMG recording technique. International 
Journal of Engineering Science and Technology, Vol.4 (2012) No.02, p.530-539

[4] M. I Ibrahimy, Md. R Ahsan, O. O Khalifa: Design and Optimization of Levenberg-Marquardt based Neural Network Classifier for EMG Signals to Identify Hand Motions. Measurement Science Review, Vol.13 (2013) No.3, p.142-151

[5] H M.Al-Angari, G Kanitz, S Tarantino, et al: Distance and mutual information methods for EMG feature and channel subset selection for classification of hand movements. Biomedical Signal Processing and Control, Vol.27 (2016) p.24-31

[6] M AbdelMaseeh, T.W Chen, and D. W Stashuk: Extraction and Classification of Multichannel Electromyographic Activation Trajectories for Hand Movement Recognition. Transactions on Neural Systems and Rehabilitation Engineering, Vol.24 (2016) No.6, p.662-673

[7] G Jang, J Kim, Y.J Choi, et al: Human Shoulder Motion Extraction Using EMG Signals. International Journal of Precision Engineering and Manufactruring, Vol.15 (2014) No.10 p.2185-2192

[8] C Cipriani, F Zaccone, S Micera, et al: On the Shared Control of an EMG-Controlled Prosthetic Hand: Analysis of User-Prosthesis Interaction. Transactions on Robotics, Vol.24 (2008) No.1 p. $170-184$

[9] F. A Omari, J Hui, C. L Mei, et al: Pattern Recognition of Eight Hand Motions Using Feature Extraction of Forearm EMG Signal. Proceedings of the National Academy of Sciences, Vol.84 (2014) No.3 p.473-480

[10]C Castellini, P V D Smagt: Surface EMG in Advanced Hand Prosthetics. Biological Cybernetics, (2009) No.100 p.35-47 